\title{
Superluminous Supernovae
}

\author{
Robert M. Quimby \\ Kavli Institute for the Physics and Mathematics of the Universe \\ Todai Institutes for Advanced Study, University of Tokyo \\ 5-1-5 Kashiwa-no-Ha, Kashiwa City, Chiba 277-8583, Japan \\ email: robert.quimby@ipmu.jp
}

\begin{abstract}
Not long ago the sample of well studied supernovae, which were gathered mostly through targeted surveys, was populated exclusively by events with absolute peak magnitudes fainter than about -20 . Modern searches that select supernovae not just from massive hosts but from dwarfs as well have produced a new census with a surprising difference: a significant percentage of supernovae found in these flux limited surveys peak at -21 magnitude or brighter. The energy emitted by these superluminous supernovae in optical light alone rivals the total explosion energy available to typical core collapse supernovae $\left(>10^{51} \mathrm{erg}\right)$. This makes superluminous supernovae difficult to explain through standard models. Adding further complexity to this picture are the distinct observational properties of various superluminous supernovae. Some may be powered in part by interactions with a hydrogen-rich, circumstellar material but others appear to lack hydrogen altogether. Some appear to be powered by large stores of radioactive material, while others fade quickly and have stringent limits on $56-\mathrm{Ni}$ production. In this talk I will discuss the current observational constrains on superluminous supernova and the prospects for revealing their origins.
\end{abstract}

Keywords. supernovae: general

Until nearly the turn of the century, the sample of supernovae discoveries with peak absolute magnitudes brighter than about -19 in the optical was dominated by Type Ia events, only a handful of supernovae connected to the deaths of massive stars were observed to cross this threshold, and there were no well studied supernovae brighter than -21 magnitude. Richardson et al. (2002) studied the peak magnitude distributions of supernovae including events listed in the Asiago Supernova Catalog (Barbon et al. 1999) for which only minimal discovery reports were available. A few events were noted as possibly brighter than -21 magnitude, although most of these can likely be explained by calibration errors $\dagger$.

The absolute magnitude distribution expected for a local, volume limited survey is shown in Fig. 1. None of the supernovae in the volume limited samples of the Lick Observatory Supernova Search (LOSS) have peak magnitudes brighter than -20 (Li et al. 2011). A flux limited survey was conducted by the Supernova Cosmology Project in the Spring of 1999, which perhaps netted the first high luminosity $(M<-21)$ supernova discovery, SN 1999as (Deng et al. 2001). However, it would be several more years before the first unambiguous superluminous supernova (SLSN) was detected and confirmed by multiple research groups.

SN 2006gy proved to be a rather surprising event. At just $72 \mathrm{Mpc}$, this supernova was observed to peak at about 14th magnitude-bright enough to be studied with even small aperture telescopes or photon hungry instruments on the larger ones-or an absolute

$\dagger$ For example, the reported magnitude for SN 1988 O places it near -22 mag absolute, but it was spectroscopically connected to the subluminous Type Ia class (Branch et al. 1993), and the magnitude given in IAUC 4601 was likely incorrect (J. Mueller, priv. comm.). 


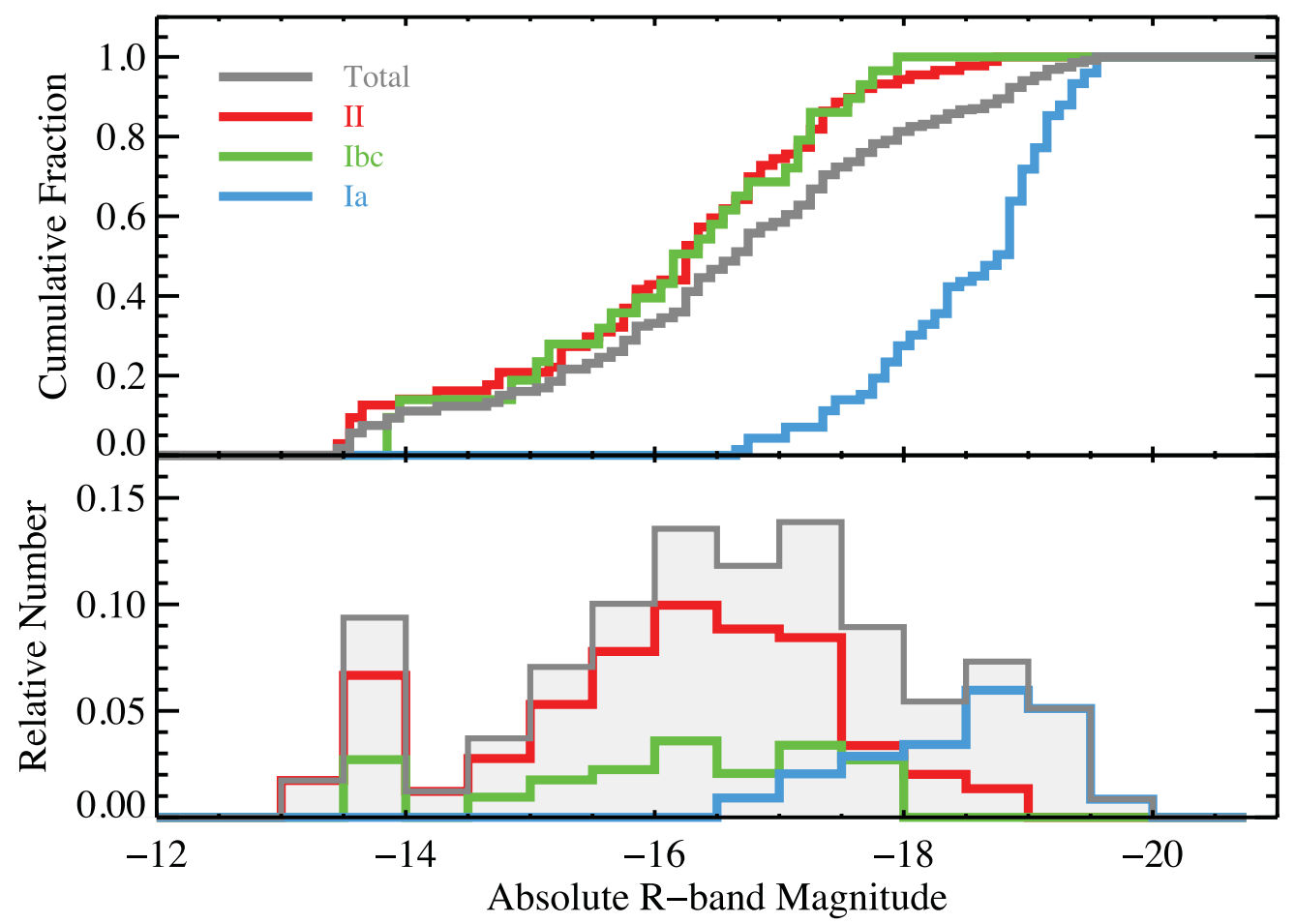

Figure 1. Peak R-band magnitude distribution for supernovae in an local, volume limited sample. Based on data from the Lick Observatory Supernova Search (Li et al. 2011).

magnitude of nearly -22 after extinction correction. And SN 2006gy did not just peak at 100 times the optical luminosity of a typical supernova, it remained brighter than normal Type Ia supernovae for 5 months, and brighter than any other well studied supernovae for 3 months (e.g. Smith et al. 2007; Ofek et al. 2007). Integration of the light curve shows the energy radiated in photons exceeds $10^{51} \mathrm{erg}$. Just how this energy was generated and how such a massive star (likely $M_{\mathrm{MS}}>100 M_{\odot}$ ) came to be in the core of a giant, metal rich galaxy, remain active research topics.

In any case, SN 2006gy clearly demonstrated that high luminosity supernovae can exist in the local universe, and this realization helped in the discovery of additional SLSNe. In particular, SN 2005ap had (obviously) been detected earlier, but it was difficult to accept the consequences of its spectroscopically determined redshift. At $z=0.28$, SN 2005ap was possibly even more luminous than SN 2006gy (Quimby et al. 2007; a definitive statement remains difficult given the unfiltered nature of the light curve). The spectra (see Fig. 2) are similar to SN 2006gy in the sense that the strong P-Cygni profiles evident in normal supernova are lacking, leaving mainly featureless blue continua, but with an important difference: SN 2006gy shows a complex H $\alpha$ profile (Smith et al. 2010), while SN 2005ap appears to lack hydrogen all together. SN 2005ap also shows a series of broad absorption dips near rest frame $4200 \AA$ (identified as OII; Quimby et al. 2011), which are not present in the spectra of SN 2006gy.

Another SLSN detected by the Robotic Optical Transient Search Experiment (ROTSEIIIb; Akerlof et al. 2003) supernovae searches shows further spectroscopic differences. Spectra of SN 2008es shows a broad HeII $\lambda 4686$ line early on and broad $\mathrm{H} \alpha$ develops later, but the narrow emission lines such as those present in SN 2006gy are not observed 


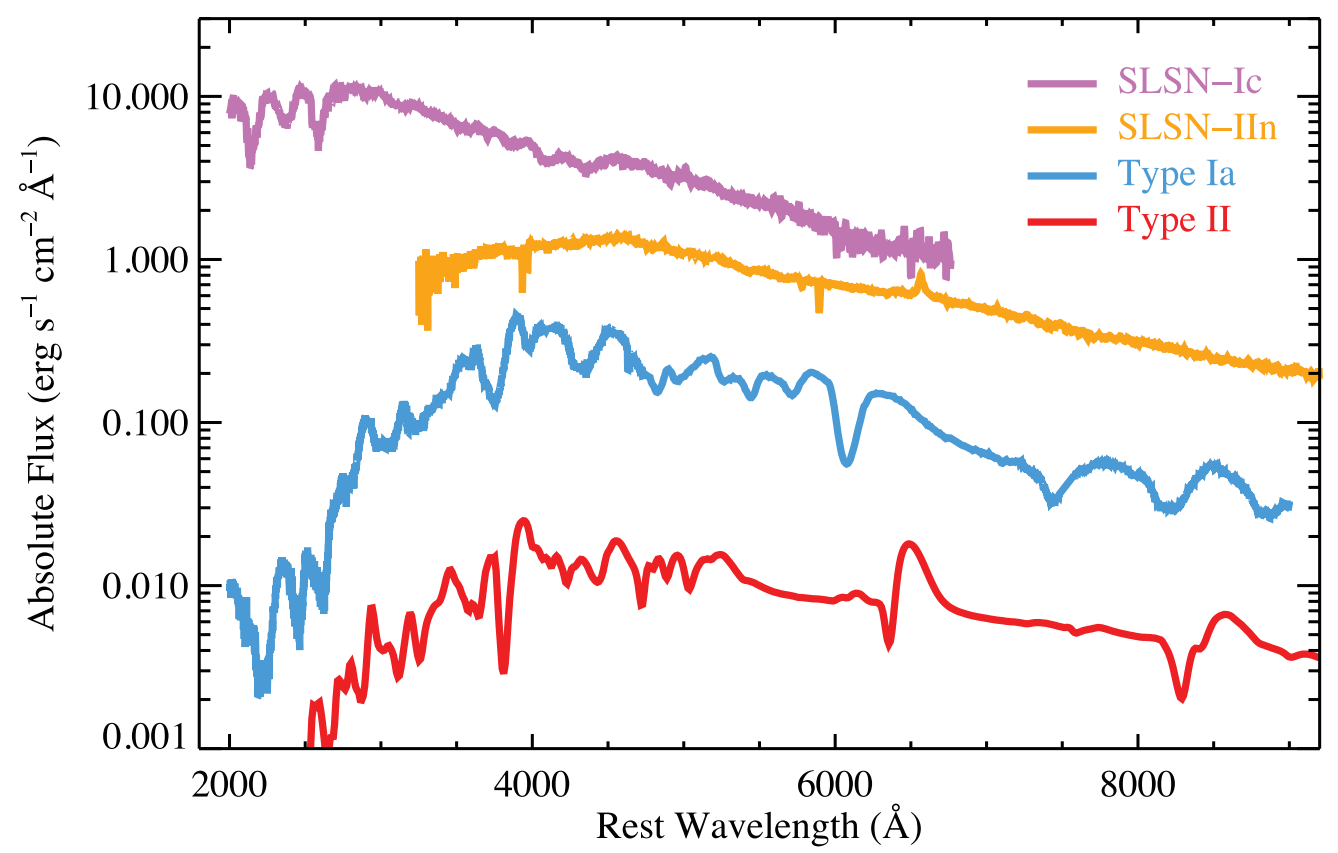

Figure 2. Spectra of normal and superluminous supernovae taken near peak (optical) brightness. The SLSN-Ic spectrum is a composite of SCP06F6 (Barbary et al. 2009), PTF09cnd (Quimby et al. 2011), and SN2005ap (Quimby et al. 2007), the SLSN-IIn is SN 2006gy from Smith et al. (2007), the Type Ia is a combination of SN 1992A (Kirshner et al. 1993) and SN 2003hv (Leloudas et al. 2009), and the Type II is a Nugent template (see supernova.lbl.gov/ nugent/nugent_templates.html). Flux values have been scaled to typical values for each class. SLSNe are about 10 times brighter than typical Type Ia supernovae in the optical, but in the UV, they can be a thousand times more luminous.

(Miller et al. 2009; Gezari et al. 2009). SN 2008es was also the first SLSNe to be studied in the UV with the Swift (Gehrels et al. 2004) Ultraviolet/Optical Telescope (UVOT; Roming et al. 2005). These data constrain the broad band spectral energy distribution and its time evolution, which can be fit relatively well with a cooling black body. The temperatures derived from this fit show that SN 2008es stayed rather hot for over a month even as the photosphere expanded at $\sim 10000 \mathrm{~km} \mathrm{~s}^{-1}$. The lack of adiabatic cooling thus suggest a large initial radius at (or beyond) the limits of super red giant envelopes.

The Palomar Transient Factory (PTF; Law et al. 2009; Rau et al. 2009) began discovering a steady supply of SLSNe from its commissioning in 2009. Some of the first of these, PTF09atu, PTF09cnd, SN 2009jh, and SN 2010gx were found to be hydrogen poor SLSNe similar to SN 2005ap (SLSN-Ic; Quimby et al. 2011; see also Pastorello et al. 2010). These supernovae were also followed-up in the UV using Swift. Like SN 2008es, there is only slow cooling over time even as the ejecta expand at $>10000 \mathrm{~km} \mathrm{~s}^{-1}$. Unlike SN 2008es, however, these events lack hydrogen. There are no stripped envelop progenitor systems known (to the author at least) with bound, hydrogen poor material distributed at $\sim 10^{15} \mathrm{~cm}$, which suggests an unbound configuration.

Prior to the start of the PTF survey proper, a "dry run" was conducted to test the follow-up paradigm that would come to characterize the future survey. This resulted in the intensive follow-up of SN 2007bi (Gal-Yam et al. 2009). The spectra of SN 2007bi lack hydrogen (and strong SiII $\lambda 6355$ ), and it can thus be classified as a SLSN-Ic. However, 


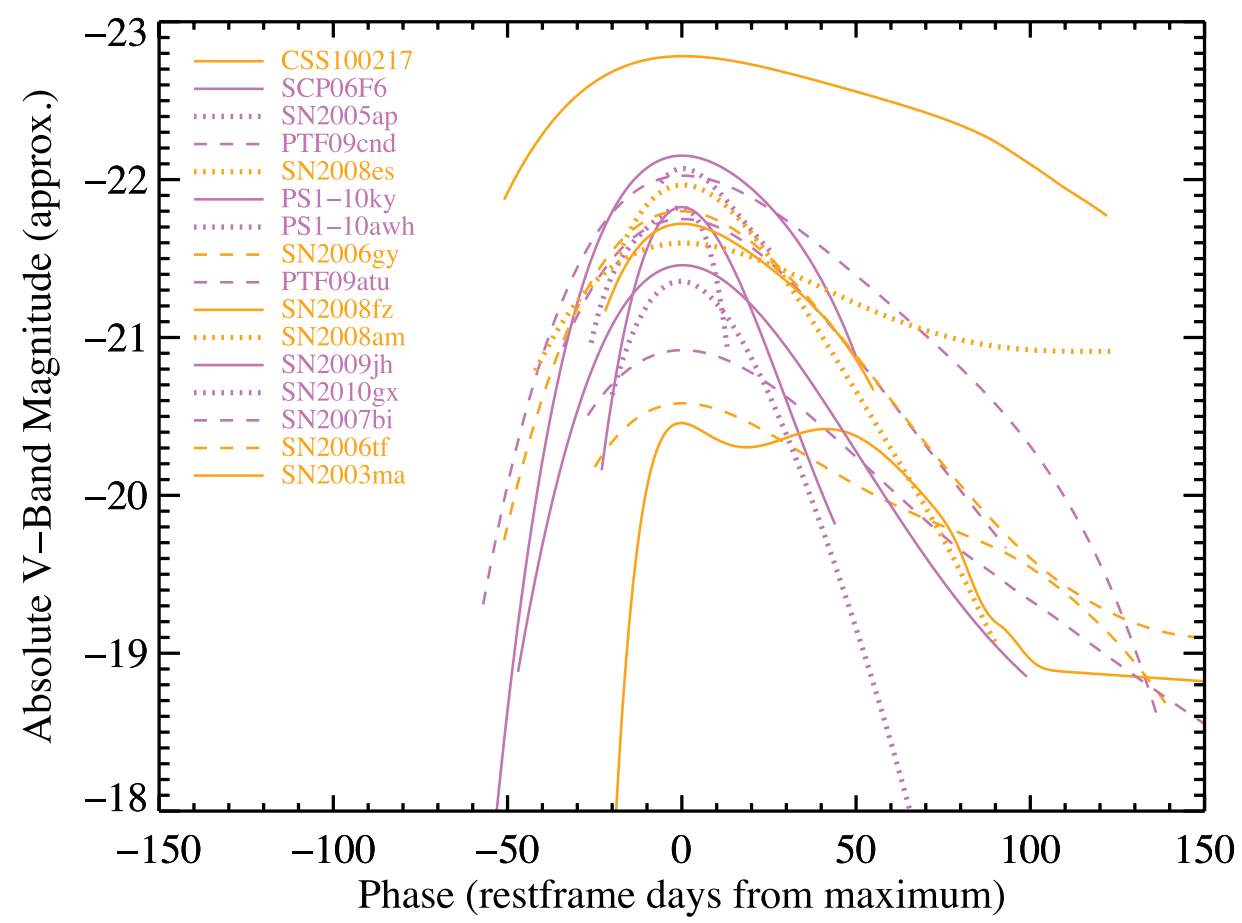

Figure 3. Approximate light curves in the rest frame, absolute V-band magnitudes for a collection of published SLSNe, including the SLSNe candidate, CSS100217. Data adapted from Drake et al. 2011, Barbary et al. 2009, Quimby et al. 2007, Quimby et al. 2011, Gezari et al. 2009, Miller et al. 2009, Chomiuk et al. 2011, Smith et al. 2007, Drake et al. 2010, Pastorello et al. 2010, Gal-Yam et al. 2009, Smith et al. 2008, and Rest et al. 2011. The light curve of SN 2006tf is supplemented with unpublished ROTSE-IIIb observations. Note that for some objects (e.g. SN 2005ap) only unfiltered data are available, and for others (e.g. SCP06F6) the available pass bands do not sample the rest frame V-band. This and the simplistic k-corrections used may lead to systematic errors of up to a few tenths of a magnitude.

there is a key difference from the SN 2005ap-like SLSNe-Ic: SN 2007bi shows photometric and spectroscopic evidence for a large ${ }^{56} \mathrm{Ni}$ yield while this is excluded for the SN 2005aplike events (Quimby et al. 2011). In particular, the light curve of SN 2007bi fades by about 1 magnitude every 100 days, as expected from the decay of ${ }^{56} \mathrm{Ni}$ 's daughter product, ${ }^{56} \mathrm{Co}$ into ${ }^{56} \mathrm{Fe}$. Other SLSN-Ic like PTF09cnd fade more rapidly and limit the production of ${ }^{56} \mathrm{Ni}$ to $\lesssim 1 M_{\odot}$. The light curves of various SLSN-Ic and hydrogen rich SLSN-II are shown in Fig. 3.

Some SLSNe, like SN 2007bi for example, may be powered mainly by radioactive decay ${ }^{56} \mathrm{Ni}$, but others (e.g. PTF09cnd) reach peak luminosities too great to be explained by the ${ }^{56} \mathrm{Ni}$ production allowed by their late time photometric limits. Some SLSNe, like SN 2006gy for example, may be powered mainly by interactions of the SN ejecta with pre-SN winds, but others (e.g. SN 2008es) show no obvious signs of such ongoing interactions. It may therefore be that there are fundamentally different engines powering these observationally distinct events. On the other hand, the principle sources of power may yet be related and it is stochastic differences in the final years of the progenitors that affect the observations.

A possible process connecting the engines powering at least some SLSNe is the conversion of kinetic energy in the supernova ejecta into radiant energy via interaction with 
slower moving material. This is most clearly evident in SLSNe-IIn such as SN 2006gy, where the narrow emission features require slow moving material in the vicinity of the explosion (fast moving material would give rise to broad, not narrow emission features). It is possible that events like SN 2008es also derive some of their power from ejecta/CSM interactions, but in this case the distribution of CSM must be fast moving or truncated such that the slow moving material has mostly been overtaken by the SN ejecta by the time the spectroscopic observations begin. Extending this model further, if the CSM was depleted of its hydrogen (for example, if the progenitor was striped of its hydrogen long before the SN explosion), the ejecta/CSM interaction could in principle provide a similar transfer of kinetic energy into photons. A possible source for such hydrogen poor CSM may be material cast off by instabilities in the cores of very massive stars in their final years (e.g. Woosley et al. 2007; Umeda \& Nomoto 2008).

Another possibility is that the high luminosities are achieved by thermalization of energy deposited into an expanding SN envelope by a compact remnant that formed as a result of the core-collapse. In the magnetar model (Kasen \& Bildsten 2010; Woosley 2010), rotational energy from the nascent neutron star is transferred (by an unspecified process) to the ejecta mass. Kasen \& Bildsten 2010 show that such models can reproduce at least the light curves of events like SN 2008es and even SN 2007bi with plausible initial rotation periods and magnetic field strengths. In this case, the progenitors could be of more modest initial masses.

We can get some insights into the progenitors by studying the broader environments in which these SLSNe explode (e.g. their hosts). Neill et al. 2011 have studied the NUVOptical color vs. Optical magnitude distribution of a number of high luminosity supernovae and they find a preference for fainter, bluer hosts when compared to the broader population of GALEX to SDSS matched galaxies. However, the sample studied is still consistent with the giant to dwarf host distribution of normal luminosity core-collapse supernovae from PTF (Arcavi et al. 2010).

Looking at the SLSNe samples from ROTSE-IIIb and PTF, there is not an obvious preference among SLSNe-IIn for dwarf or giant hosts (both surveys find SLSNe-IIn in hosts of various luminosities, faint to bright). However, the SLSN-Ic do appear to prefer dwarf host galaxies. There is possibly only one SLSN-Ic out of more than a dozen discoveries that is hosted by a giant.

The rates of SLSNe can also offer some constraints on the progenitor systems when the birth rates of such progenitors are known. Based on the ROTSE-IIIb sample, preliminary results (Quimby et al. in prep.) suggest that there is one SLSN (of any type) for about every 1000 core-collapse supernova in the local $(z \sim 0.2)$ universe. If we assume that the distribution of SLSN-Ic is skewed brighter than the SLSN-II distribution, as hinted at by the ROTSE-IIIb sample at least, then this would imply that most SLSN have hydrogen, since ROTSE-IIIb has found a roughly equal number of each while the SLSN-Ic would be drawn from a larger volume in this case. The SLSN-Ic rate would then be about one for every $10^{4}$ core-collapse supernovae.

This is still a sufficiently high rate, and the SLSNe are luminous enough that it should be possible with existing instruments to detect SLSNe out to very high redshifts (e.g. $z \sim 4$ with Subaru). This opens the possibility of using SLSNe to glean insights into the distant universe. First of all, if the SLSNe are connected to the most massive stars (as seems to be the case at least for SN 2006gy), then their rates should evolve with redshift with the cosmic star formation history. If there are changes in the IMF such as a "top-heavy" IMF at higher redshifts, then we could expect more SLSNe per unit star formation. Thus checking if the distant to local SLSN rate differs from the distant to local SFR could be one way to search for evolution in the IMF. Additionally, absorption 
features imprinted in the otherwise smooth continua of SLSNe could carry information about the chemistry of distant stellar nurseries. Considering the coming suite of wide field cameras on moderate to large aperture telescopes, the future of SLSNe research appears, well, bright.

\section{References}

Akerlof, C. W., Kehoe, R. L., McKay, T. A., et al. 2003, PASP, 115, 132

Arcavi, I., Gal-Yam, A., Kasliwal, M., et al. 2010, ApJ, 721, 777

Barbary, K., Dawson, K. S., Tokita, K., et al. 2009, ApJ, 690, 1358

Barbon, R., Buondí, V., Cappellaro, E., \& Turatto, M. 1999, A\&A (Supplement), 139, 531

Branch, D., Fisher, A., \& Nugent, P. 1993, AJ, 106, 2383

Chatzopoulos, E., Wheeler, J. C., Vinko, J., et al. 2011, ApJ, 729, 143

Chomiuk, L., Chornock, R., Soderberg, A. M., et al. 2011, ApJ, 743, 114

Deng, J. S., Hatano, K., Nakamura, T., et al. 2001, in Astronomical Society of the Pacific Conference Series, Vol. 251, New Century of X-ray Astronomy, ed. H. Inoue \& H. Kunieda, 238

Drake, A. J., Djorgovski, S. G., Mahabal, A., et al. 2011, ApJ, 735, 106

Drake, A. J., Djorgovski, S. G., Prieto, J. L., et al. 2010, ApJ, 718, L127

Gal-Yam, A., Mazzali, P., Ofek, E. O., et al. 2009, Nature, 462, 624

Gehrels, N., Chincarini, G., Giommi, P., et al. 2004, ApJ, 611, 1005

Gezari, S., Halpern, J. P., Grupe, D., et al. 2009, ApJ, 690, 1313

Kasen, D. \& Bildsten, L. 2010, ApJ, 717, 245

Kirshner, R. P., Jeffery, D. J., Leibundgut, B., et al. 1993, ApJ, 415, 589

Law, N. M., Kulkarni, S. R., Dekany, R. G., et al. 2009, PASP, 121, 1395

Li, W., Leaman, J., Chornock, R., et al. 2011, MNRAS, 413

Miller, A. A., Chornock, R., Perley, D. A., et al. 2009, ApJ, 690, 1303

Neill, J. D., Sullivan, M., Gal-Yam, A., et al. 2011, ApJ, 727, 15

Ofek, E. O., Cameron, P. B., Kasliwal, M. M., et al. 2007, ApJ, 659, L13

Pastorello, A., Smartt, S. J., Botticella, M. T., et al. 2010, ApJ, 724, L16

Quimby, R. M., Aldering, G., Wheeler, J. C., et al. 2007, ApJ, 668, L99

Quimby, R. M., Kulkarni, S. R., Kasliwal, M. M., et al. 2011, Nature, 474, 487

Rau, A., Kulkarni, S. R., Law, N. M., et al. 2009, PASP, 121, 1334

Rest, A., Foley, R. J., Gezari, S., et al. 2011, ApJ, 729, 88

Richardson, D., Branch, D., Casebeer, D., et al. 2002, AJ, 123, 745

Roming, P. W. A., Kennedy, T. E., Mason, K. O., et al. 2005, Space Sci. Revs, 121, 95

Smith, N., Chornock, R., Li, W., et al. 2008, ApJ, 686, 467

Smith, N., Chornock, R., Silverman, J. M., Filippenko, A. V., \& Foley, R. J. 2010, ApJ, 709, 856

Smith, N., Li, W., Foley, R. J., et al. 2007, ApJ, 666, 1116

Umeda, H. \& Nomoto, K. 2008, ApJ, 673, 1014

Woosley, S. E. 2010, ApJ, 719, L204

Woosley, S. E., Blinnikov, S., \& Heger, A. 2007, Nature, 450, 390

\section{Discussion}

KATZ: What are the upper limits for X-ray emission from the SLSNe?

QuimBY: The only SLSN I know of with a reported X-ray detection is SN 2006gy (Smith et al. 2007), and not everyone agrees that this is actually a detection. Upper limits exist for an additional number of SLSN-IIn (e.g. Chatzopoulos et al. 2011) and SLSN-Ic (e.g. Quimby et al. 2011), but these are not often all that constraining given the typically 
large distances: the limits near optical maximum are typically around $10^{43} \mathrm{erg} \mathrm{s}^{-1}$ in the Swift/XRT band, which is lower than the optical luminosities.

GAL-YAM: I know of no detections in X-ray, including, in my opinion, SN 2006gy.

MEtzGer: How do the host galaxies of SLSN-Ic compare to those of GRB hosts?

QuimbY: The sample of SLSN-Ic hosts is still small, but it would appear that SLSN-Ic may favor even lower luminosity hosts than do GRBs. 